\title{
Fast Neutron and Gamma-Ray Interrogation of Air Cargo Containers
}

\author{
J.E. Eberhardt, Y. Liu, S. Rainey, G.J. Roach, R.J. Stevens, B.D. Sowerby* and \\ J.R. Tickner \\ CSIRO Minerals, Private Mail Bag 5, Menai NSW 2234 Australia \\ E-mail: brian. sowerby@csiro.au
}

There is a worldwide need for improved methods for the scanning of consolidated air cargo for contraband such as illicit drugs and explosives. The strong preference is to directly image the contents of cargo containers without unpacking and with scan times of less than a few minutes per container. In the current paper the various alternative X-ray, gamma-ray and neutron techniques will be discussed and compared.

The Commonwealth Science and Industrial Research Organisation (CSIRO) has been working with the Australian Customs Service to develop a scanner capable of directly scanning airfreight containers in 1-2 minutes. The scanner combines fast neutron and gamma-ray radiography (FNGR) to provide high-resolution images that include information on material composition. The composition information greatly facilitates the task of image analysis. A commercial-scale scanner has been installed at an Australian Customs Service facility at Brisbane International Airport to trial the technology and associated business processes. The trial will continue for up to 18 months and includes a comprehensive evaluation by an independent team leader engaged by Customs. The scanner facility incorporates an extensive automated cargo handling system, making possible the continuous processing of large numbers of containers.

Results are presented on the image quality of the FNGR scanners, their dynamic range and their ability to discriminate different material compositions. Some results from the FNGR scanner are presented, highlighting the ability of the method to distinguish ill-defined, low-density organic materials that are difficult to detect using conventional X-ray scanners.

International Workshop on Fast Neutron Detectors

University of Cape Town, South Africa

April 3-6, 2006 


\section{Industry Requirements}

There is a worldwide need for effective mass screening of cargo containers at airports, seaports and road border crossings. The main objectives are the detection of contraband such as illicit drugs, explosives, nuclear materials and weapons, and the verification of declared manifests. Mass screening by manual inspection of air cargo containers is generally not practical, primarily because of time constraints and the high labour requirements for unpacking, inspecting and repacking cargo. There is therefore a critical need for improved air cargo mass screening inspection systems. Due to the large number of cargo containers that require inspection it is critical that any scanning system should be capable of working on consolidated cargo, taking at most a few minutes per container. The requirements for the various types of cargo (loose, palletised and containerised) differ, mainly due to cargo characteristics (mass, thickness, uniformity). In addition, the various types of contraband have different requirements regarding the acceptable incidence of false negatives and false positives. In the present paper technologies for the screening of air cargo are discussed.

In the case of air cargo, packages or goods are packed inside lightweight aluminium air cargo containers (Unit Load Devices (ULDs)) or on pallets with an average bulk density of up to $0.4 \mathrm{~g} \mathrm{~cm}^{-3}$. The most common ULDs have a width of about $1.6 \mathrm{~m}$ and a maximum weight of 1.6 tonnes. However some weigh up to 6.8 tonnes and are up to 2.44 metres wide. Achieving a satisfactory level of mass screening of air cargo is difficult. Complexities include the sheer volume of cargo, the time-critical nature of the movement of cargo, and the variation in cargo presentation for transport (e.g. loose, consolidated, palletised, shrink-wrapped).

The requirements for a mass scanning system for air cargo can be summarised as follows:

- It must produce high resolution images that are readily interpreted

- It must accurately scan consolidated cargo without unpacking

- Scan times should be less than about 2 minutes per container and turnaround time should be minimised

- It must comply with strict radiation safety requirements for both operating staff and cargo

- False indications should be minimised

- It needs to be of acceptable cost, capable of constant operation and be easy to maintain.

\section{Current and Emerging Technologies}

\subsection{X-Ray and Gamma-Ray Methods}

Only fast (high-energy) neutrons and high-energy X-rays or gamma rays have the required penetration for imaging the contents of cargo containers. There has been a significant amount of work performed over the past decade on a range of X-ray, gamma-ray and neutron-based inspection techniques particularly for the detection of explosives in aircraft luggage, etc. This work has led to the development of sophisticated X-ray imaging systems that are now the 
industry standard for scanning in the aviation industry. These X-ray systems can provide high resolution 2-D density images of the contents of containers and are ideally suited to the detection of metallic objects with readily identifiable shapes such as firearms and other weapons. They are not well suited to the detection of illicit substances that have similar densities and shapes to many common substances. Image quality is crucial and recent developments have focussed on improving the resolution and contrast of the collected images.

There are many interesting new developments in X-ray and gamma-ray techniques. One of particular interest is dual-energy $\mathrm{X}$-ray radiography at high energies (up to about $10 \mathrm{MeV}$ ) [1]. This technique has the potential to extend the applications of convential dual-energy X-ray radiography (widely used for luggage screening) to cargo. However the technique has limited ability to distinguish various classes of organic materials.

\subsection{Neutron Techniques}

For neutron techniques to be successfully applied to cargo screening they must firstly meet the aviation security and border protection specialists requirements listed above and secondly they must have significant advantages over the established and developing X-ray techniques. Fast neutron techniques are attractive for these applications as they have the required penetration, they interact with matter in a manner complementary to X-rays and they can be used to determine elemental composition.

Neutron interrogation techniques fall into two categories, namely radiography systems and systems that measure secondary radiation (neutrons or gamma rays). Fast neutron radiography techniques have the advantage of direct measurement of transmitted neutrons and are therefore more efficient (and therefore require lower neutron source strengths) than techniques measuring secondary radiation such as neutron-induced gamma rays. There have been a number of excellent general reviews of neutron techniques for non-intrusive inspection [2,3]. In the present paper, discussion is confined to neutron radiography techniques and applications.

Although neutron radiography techniques have been around for many years, it is only recently that they have been investigated for cargo inspection. A fast neutron and gamma ray and radiography system has been developed [4] to supplement the Ancore Pulsed Fast Neutron Analysis (PFNA) system. In this system nanosecond-pulsed fast neutrons and gamma rays from an accelerator are transmitted through the object and the detected neutron and gamma ray signals are separated by arrival time.

Various groups have investigated fast neutron resonance radiography to determine elemental composition $[5,6,7]$. However these methods require the use of a high energy particle accelerator. 


\section{Fast Neutron and Gamma Radiography}

In the present paper we describe an alternative fast neutron and gamma ray radiography technique that utilises monoenergetic $14 \mathrm{MeV}$ neutrons from a commercially available sealed tube neutron generator. The use of a simpler source offers a significant advantage for airport applications. The CSIRO Fast Neutron and Gamma Radiography (FNGR) technique forms 2dimensional projection images showing both density (strictly, mass per unit area) and composition. It works by measuring the attenuation, through the object to be imaged, of beams of fast neutrons and gamma rays from the radiation sources to an array of detectors.

Assuming a narrow beam geometry in which scattered radiation does not reach the detector, the transmission of fast neutrons through an object of density $\rho$ and thickness $x$ can be calculated using the equation:

$$
I_{n} / I_{n}^{0}=\exp \left(-\mu_{n} \rho x\right)
$$

where $I_{n}$ is the measured neutron intensity through the object, $I_{n}^{0}$ is the measured neutron intensity with the object removed and $\mu_{n}$ is the neutron mass attenuation coefficient. Similarly gamma-ray transmission can be written as:

$$
I_{g} / I_{g}^{0}=\exp \left(-\mu_{g} \rho x\right)
$$

where $I_{g}$ is the measured gamma-ray intensity through the object, $I_{g}^{0}$ is the measured gamma-ray intensity with the object removed and $\mu_{g}$ is the gamma mass attenuation coefficient.

The ratio, $R$, of the neutron and gamma-ray attenuation coefficients is:

$$
R=\frac{\mu_{n}}{\mu_{g}}=\frac{\ln \left(I_{n} / I_{n}^{0}\right)}{\ln \left(I_{g} / I_{g}^{0}\right)}
$$

$R$ can therefore be determined directly from the measured neutron and gamma-ray transmissions without knowing the mass of material in the radiation beams. This ratio provides a powerful discriminator between different classes of material. Figure 1 illustrates calculated values of $R$ for a range of materials using $14 \mathrm{MeV}$ fast neutrons and ${ }^{60} \mathrm{Co}$ gamma-rays (energies 1.17 and $1.33 \mathrm{MeV})$.

The accurate measurement of $R$ would in principle allow a wide variety of material classes to be directly identified. However, as the neutron and gamma-ray beam transmissions are measured along a line between the radiation source and detector, the measured $R$ value is an average for the materials in front of each detector element. In simple cases, correction can be made for overlying or underlying materials to allow the $R$ value of an individual object to be determined. 


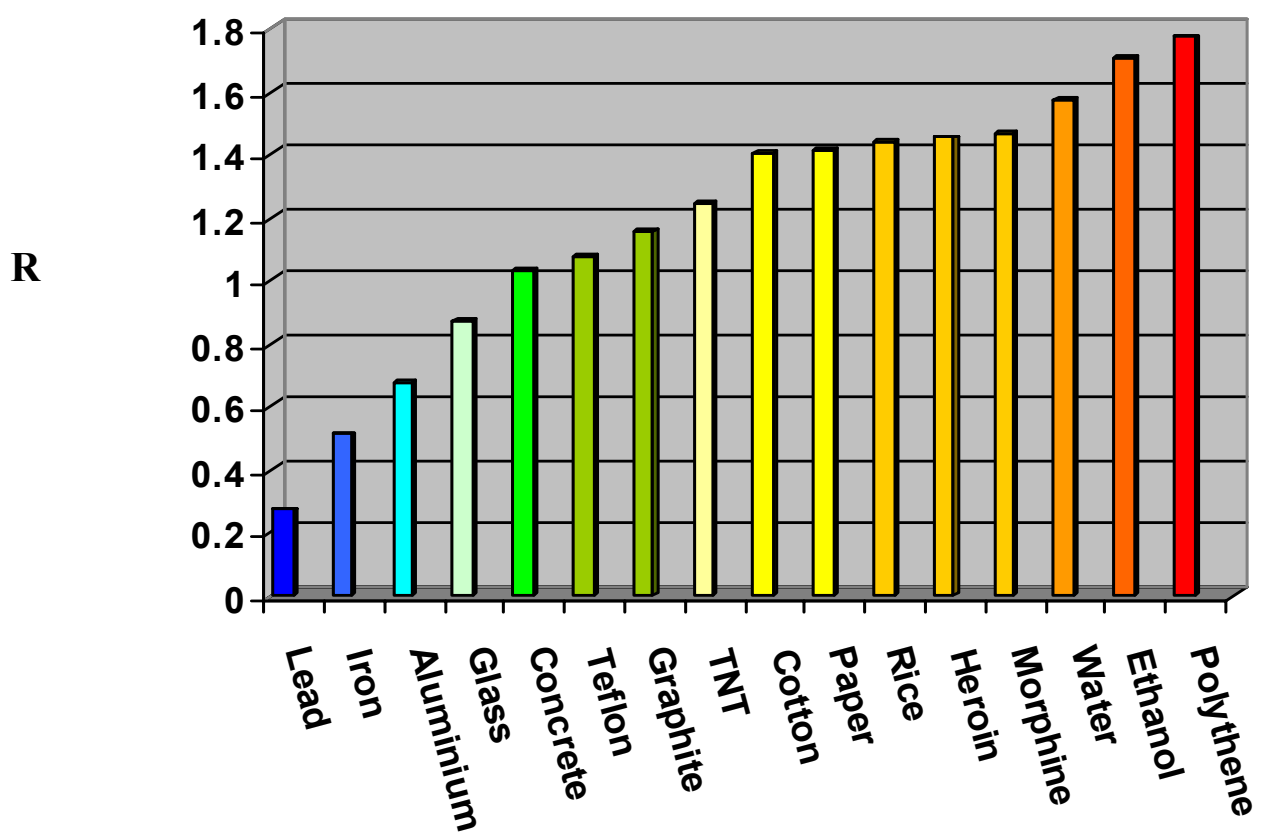

Figure 1. Calculated $\mathrm{R}$ values for a range of materials, using $14 \mathrm{MeV}$ neutrons and ${ }^{60} \mathrm{Co}$ gamma rays.

\section{CSIRO Air Cargo Scanner at Brisbane Airport}

An early prototype FNGR scanner has been described previously [8]. Based on the successful demonstration of this scanner, a commercial-scale scanner has been developed and installed in an Australian Customs Service facility at Brisbane International Airport so that the technology and associated business processes can be trialed in a real time operational environment.

The radiation sources are mounted inside shielding which provides radiological protection and are located on one side of a shielded tunnel of dimensions $2.7 \mathrm{~m}$ wide and $2.7 \mathrm{~m}$ high with the $4.3 \mathrm{~m}$ high detector array on the opposite side (Figure 2). The cargo to be scanned passes through a tunnel on a chain conveyor. The radiation sources are approximately $6.1 \mathrm{~m}$ from the detector array. Collimators set into the shielding are used to accurately define the neutron and gamma-ray radiation beams. Careful design of these collimators serves to minimize radiation scattering that otherwise reduces both the resolution and contrast of the transmission images.

The main requirements on both the neutron and gamma-ray sources are intense output to allow rapid scanning; high energy to penetrate thick cargos; small active volume to preserve image resolution; reasonable capital and running costs and safety considerations. The Brisbane scanner uses a Thermo MF Physics A-711 D-T neutron generator that can produce up to $10^{10}$ neutrons/second of $14 \mathrm{MeV}$ neutrons. Key advantages of a sealed tube generator over radioisotope neutron sources include the high neutron energy of $14 \mathrm{MeV}$ and that, when turned off, the source emits essentially no radiation. The gamma-ray source is a $185 \mathrm{GBq}{ }^{60} \mathrm{Co}$ 
(energies of 1.17 and $1.33 \mathrm{MeV}$ ). The advantages of ${ }^{60} \mathrm{Co}$ include its long half-life (5.3 years), the availability of high source strengths and high specific activities, and its relatively low cost. The main disadvantage of a radioisotope source is that is cannot be turned off and requires shielding at all times.

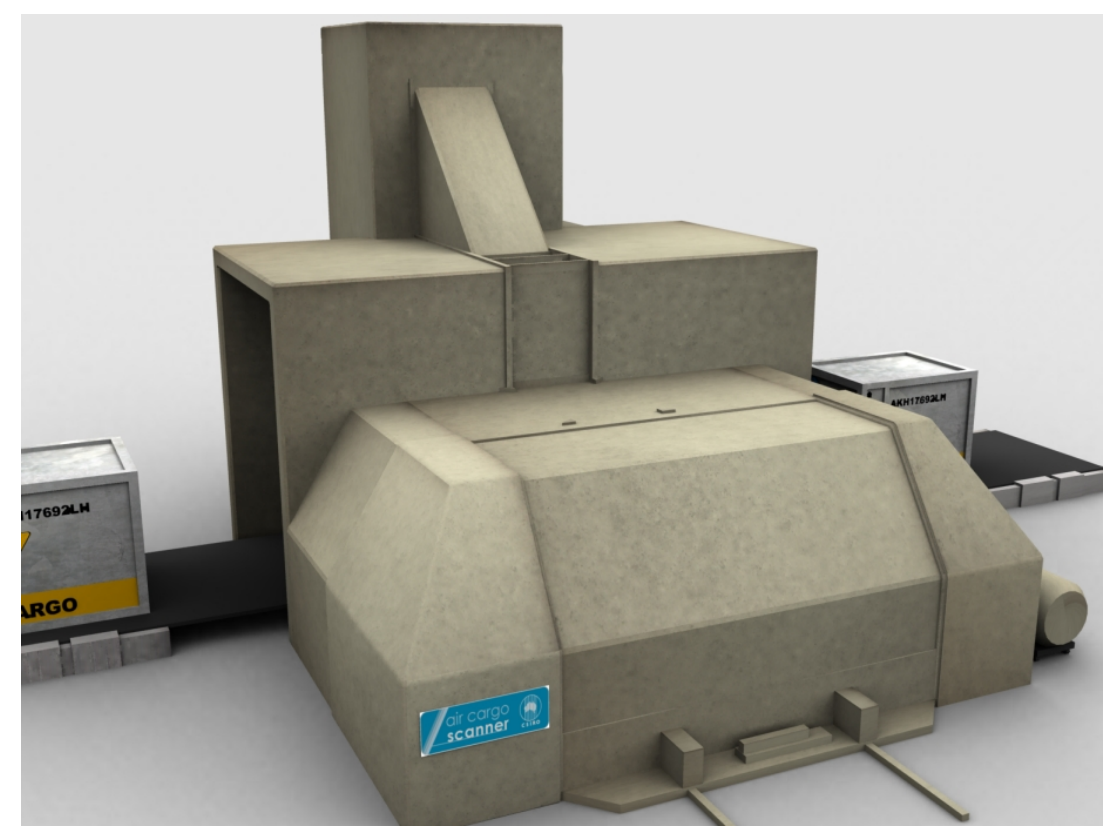

Figure 2. Model of the CSIRO Air Cargo Scanner installed in the Australian Customs facility at Brisbane airport. The source shield is shown in the foreground and the cargo tunnel and detector tower in the background.

A key aspect of the FNGR scanner was the development of high efficiency, low cost detector arrays for both fast neutrons and gamma rays. High detection efficiency was particularly important for neutrons as the brightness of conveniently available neutron sources is low. The most efficient fast neutron detectors were those based on proton recoil in plastic (or liquid) scintillators. CSIRO has developed custom-designed neutron and gamma-ray detector systems, each comprising a large number of small scintillators with photodiode readout [8]. The neutron detector system uses 704 plastic scintillator detectors and the gamma-ray detector system uses $352 \mathrm{CsI}(\mathrm{Tl})$ detectors.

Radiation levels are low because of (a) the quantity of concrete shielding used, (b) the relatively low intensity sources compared to other cargo scanners, and (c) the highly collimated fan-beams of radiation used. In addition, the scanner is located in an exclusion zone to prevent access to the scanner by unauthorized personnel. Multiple fail-safe interlock mechanisms are used to prevent people entering the scanner during its operation. The radiation levels outside the exclusion zone are less than $0.5 \mu \mathrm{Sv}$ /hour. Cargo passing through the tunnel is briefly exposed to gamma-ray and neutron beams. The total radiation dose received by the cargo during scanning is calculated to be approximately $12 \mu \mathrm{Sv}$. This is approximately the cosmic ray- 
induced natural background that would be received during about 2 hours in an aircraft at 10,000 m altitude.

As the detector system comprises narrow, vertical columns of pixels, it is necessary to move the cargo being scanned through the fan-beams of radiation to build up a complete, 2dimensional image line by line. Control and readout of the neutron and gamma-ray boards is managed via a custom-designed, high-speed serial interface. Each column of boards is daisychained together and connected to a PC. Air conditioning of the detector tower provides temperature control of the detector array to improve stability and reduce noise. The chain conveyor generates a signal for the detector readout every time the platform has traversed 5 $\mathrm{mm}$. The chain conveyor is typically operated at a speed of about $1-2 \mathrm{~m} / \mathrm{min}$ to achieve scanning times of approximately 1-2 minutes per ULD once the leading edge of the container is at the scanner.

The neutron and gamma-ray count rates in a given detector with no intervening material are approximately 800 and 23,000 counts per second respectively. Due to the higher count-rates, smaller pixel size and lower background scattering of the gamma rays, the gamma-ray image carries most of the information about shape and density. For each pixel in the image, the quantities $-\ln \left(I_{g} / I_{g}^{0}\right)$ and $R$ value are calculated. Various filters were applied to both the density and $R$ value images and corrections were made for deadtime, neutron flux variation (using a monitor detector), low count averaging and neutron and gamma cross-talk. The density and $R$ value images were combined by mapping the ratio $R$ to the hue (colour) of the pixel. In the images, low $R$ values (inorganic materials) were coloured purple or blue, intermediate $R$ values were coloured green and high $R$ values (organic materials) were coloured yellow and orange (Figure 1). This colour scheme is similar to that widely used in dual-energy X-ray scanners for luggage and parcels. The density value was used to determine the brightness (luminance) of the colour.

The user has several tools available to manipulate the displayed images including brightness and contrast controls; toggle between full-colour, black and white and inverse image displays; zoom the entire image or selected regions; and the use of histogram equalization and contouring tools. In practice, this manipulation is critical for accurately identifying subtle differences in density or composition and for detecting concealed objects.

\section{Reference Scanner}

During the development of the Brisbane scanner, CSIRO also designed and commissioned a Reference Scanner unit at the Lucas Heights Laboratory. The Reference Scanner was designed to mimic (as far as possible) the Brisbane Airport Scanner. In particular, the Reference Scanner uses the same energy neutron and gamma-ray sources, the same detector system and the same 
readout electronics and image processing software. The main differences between the two systems are:

- The Reference Scanner uses a Thermo MF Physics A-325 neutron generator which produces up to $10^{8} \mathrm{n} / \mathrm{s}$ and a $2.4 \mathrm{GBq}(88 \mathrm{mCi})$ cobalt- 60 source

- Shorter detector arrays are used in the Reference Scanner, which limits the imaged region to the bottom two thirds of a standard cargo container

- The Reference Scanner has two vertical neutron detector arrays, whereas the Brisbane Scanner has four

- The Reference Scanner uses a trolley moving on rails to transport cargo through the radiation beams, whereas the Brisbane scanner uses a chain conveyor.

The main consequence of the differences between the two systems is the speed at which cargo can be moved through the scanner. For the results presented below, the Reference Scanner was operated at a speed of $0.9 \mathrm{~m} /$ hour, meaning that around 3 hours were required to image a typical $2.5 \mathrm{~m}$ cargo compared to about 1-2 minutes in the Brisbane Scanner.

\section{Images from Reference Scanner}

Two typical scans are shown in Figures 3 and 4. Figure 3 shows the scan of a motorbike; the upper monochrome image shows only density information, such as would be obtained with an X-ray scanner and the lower image includes the composition information provided by the neutron measurement. These images provide a good indication of the overall imaging capabilities of the scanner. In particular, fine details such as the front brake cables show quite clearly, even though they are considerably smaller than the pixel size. The metal frame and engine of the bike show up blue in the composition image; whereas the fuel in the petrol tank, rubber tyres, plastic seat, lights etc show up orange. The oil in the sump (immediately above the kickstand), when averaged together with the metal in the same path, shows as a green patch.

A range of cargos were prepared to trial the reference scanner that broadly represented the types of items traveling through Australian airports. These cargos contained items such as heavy industrial machinery, electronics goods, household and office effects and fresh and preserved foodstuffs. A variety of real and simulated contraband items were concealed in these cargos which where then scanned using the reference scanner. The suspect items were detected and identified on the basis of their shape and $\mathrm{R}$ value.

A single ULD containing a wide range of items was also scanned and scans of this cargo, without concealed contraband, are shown in Figure 4. From left-to-right, the cargo contains assorted computer equipment, heavy steel industrial items, mixed boxes of food stuffs (including bottled drinks, frozen meat and fish, boxed apples) and boxes containing office files and papers. The images illustrate the spatial resolution of the scanner and its ability to distinguish between different material categories. 


\section{Summary}

CSIRO has developed the fast-neutron/gamma-ray radiography (FNGR) method for the rapid scanning of air freight containers. By combining fast-neutron and gamma-ray radiographic measurements, it is possible to produce an image that represents both the density and composition of the contents of a container. The presence of composition information greatly facilitates the task of image analysis and is particularly advantageous in the detection of lowatomic number, organic materials with ill-defined shapes such as concealed narcotics and explosives. CSIRO has installed a full-scale commercial prototype scanner at an Australian Customs Service purpose-built facility at Brisbane International Airport. Customs are undertaking a trial of the technology in a real-time working environment as well as a rigorous evaluation of the technology and associated business processes. The scanner has significant market potential and patent applications have been lodged [10].

\section{Acknowledgments}

The authors gratefully acknowledge the financial support and assistance of the Australian Customs Service and the Department of Prime Minister and Cabinet. The authors wish to thank the staff of the On-Line Analysis and Control Program and the Lucas Heights Mechanical Engineering and Electronics Groups of CSIRO Minerals for their invaluable contributions.

\section{References}

[1] D. Perion, T. Martin, S. Maitrejean, A. Frank and P. Schall, System for differentiating between organic and inorganic substances, International Patent Application WO 00/43760; 27 July 2000

[2] T. Gozani, A review of neutron based non-intrusive inspection technologies, in Conf. on Technology for Preventing Terrorism, Stanford, 12-13 March 2002.

[3] A. Buffler, Contraband detection with fast neutrons, Radiation Physics and Chemistry 71 (2004) 853

[4] J. Rynes et al, Gamma-ray and neutron radiography as part of a pulsed fast neutron analysis system, Nuclear Instruments and Methods in Physics Research A422 (1999) 895

[5] R.C. Lanza, New trends in neutron radiography, in Application of Accelerators in Research and Industry, AIP Conference Proceedings 680, eds. J.L. Duggan and I.L. Morgan, August 2003, ISBN 0-7354-0149-7

[6] H.W. Lefevre et al, Using a fast neutron spectrometer system to handle luggage for hidden explosives, Proc. Internat. Conf. On Neutrons in Research and Industry, Crete, Greece, 9-15 June 1996, SPIE Proceedings Series 2867 (1997) 206

[7] D. Vartsky, Prospects of fast neutron resonance radiography and requirements for instrumentation, these proceedings

[8] J.E. Eberhardt et al. Fast neutron radiography scanner for the detection of contraband in air cargo containers, Applied Radiation and Isotopes 63 (2005) 179

[9] D. Crnokrak et al, Fast neutron and gamma-ray detectors for the CSIRO Air Cargo Scanner, these proceedings

[10] B. D. Sowerby and J.R. Tickner, Radiographic equipment, International Patent Application No. PCT/AU03/01641, Filing date: 10 Dec 2003 

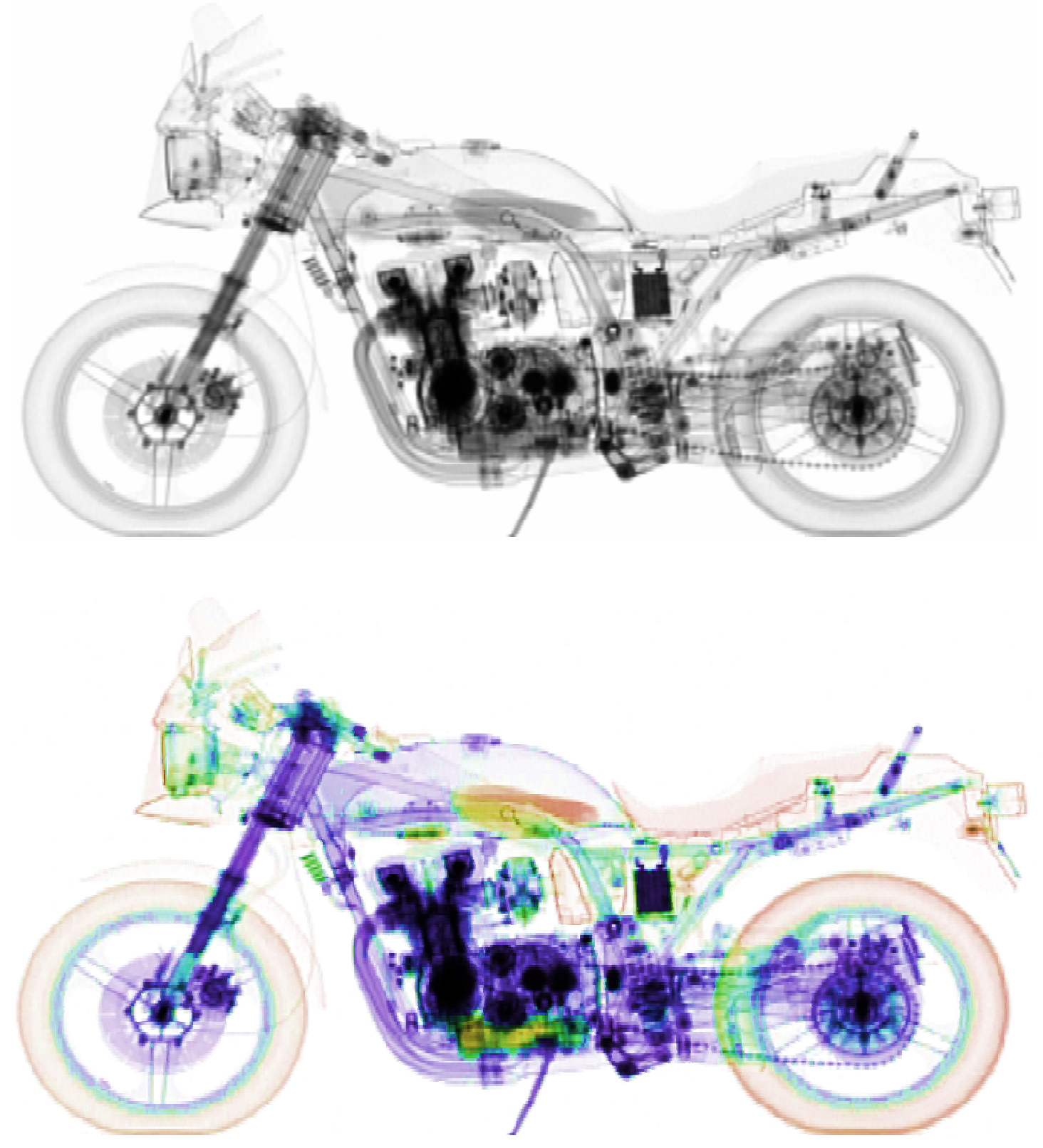

Figure 3. Gamma-ray (upper figure) and combined neutron/gamma-ray (lower figure) scans of a motorbike 

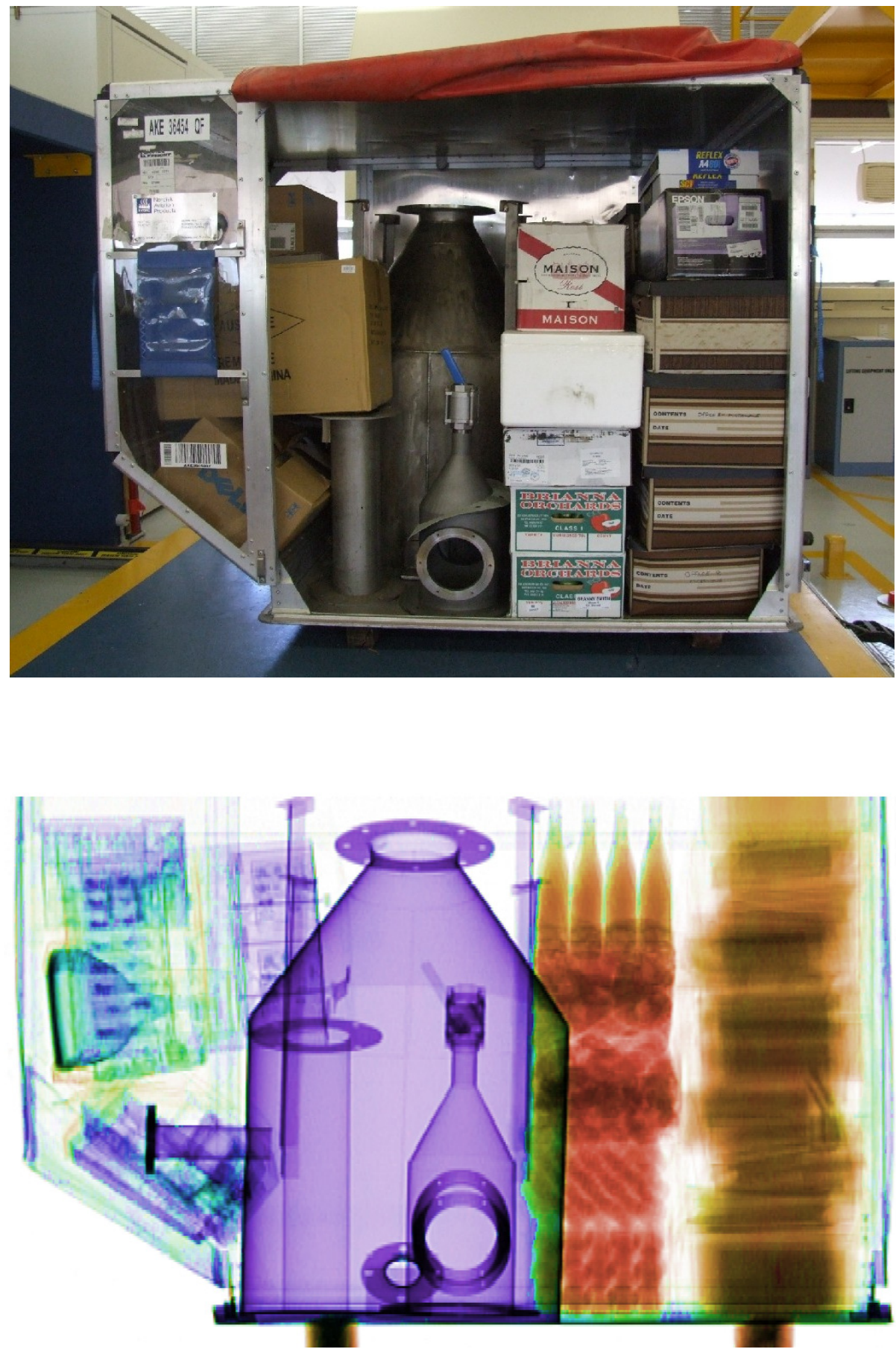

Figure 4. Photograph and combined neutron and gamma scan of ULD loaded with mixed cargo. From left-to-right, the cargo contains assorted computer equipment, heavy steel industrial items, mixed boxes of food stuffs (including bottled drinks, frozen meat and fish, boxed apples) and boxes containing office files and papers. 